\title{
Recalculation of the brightness of stars in the sensitivity band of an arbitrary receiver of radiation in the visible range
}

\author{
Alexandr Zhukov ${ }^{1,2,3,4}$, Mikhail Prokhorov ${ }^{5}$, Andrey Zakharov ${ }^{5}$, Anton Biryukov ${ }^{5}$, \\ Igor Kartsan ${ }^{1,6,7,8}$ and Vladimir Skripachev ${ }^{1,2}$ \\ ${ }^{1}$ FGBNU "Expert and Analytical Center", 33, Talalikhina St., building 4, Moscow, 109316, Russia \\ ${ }^{2}$ MIREA - Russian Technological University, 78, Vernadskogo Av., Moscow, 119454, Russia \\ ${ }^{3}$ Institute of Astronomy of the Russian Academy of Sciences, 48, Pyatnitskaya Str., Moscow, Russia \\ ${ }^{4}$ Joint Stock Company "Special Research of Moscow Power Engineering Institute", 14, Krasnokazarmennaya \\ Str., Moscow, Russia \\ ${ }_{5}^{5}$ Sternberg Astronomical Institute, Moscow State University, Universitetsky pr., 13, Moscow, 119234, Russia \\ ${ }^{6}$ Reshetnev Siberian State University of Science and Technology, 31, Krasnoiarskii Rabochii Prospekt, \\ Krasnoyarsk, 660037, Russia \\ ${ }^{7}$ Marine Hydrophysical Institute, Russian Academy of Sciences, 2, Kapitanskaya St., Sevastopol, 299011, Russia \\ ${ }^{8}$ Sevastopol State University, University str. 33, Sevastopol, 299053, Russia
}

\begin{abstract}
A procedure is described for calculating the brightness of stars in a band that does not coincide with the bands of their observation. As an example, we took the calculation for the navigation catalog of the star sensor. We present a way to reduce the orientation error by increasing the accuracy of determining the position of the photocenter of the star image on the radiation receiver - a charge-coupled matrix device. The most difficult is the accounting of the shadow signal. First, the rates of thermogeneration strongly depend on temperature. Second, the scatter of average values of thermogeneration rates in individual elements is many times greater than the random scatter (noise) in each of them. Thirdly, in addition to "normal" elements in the charge-coupled matrix device there are "hot" elements, the rates of thermogeneration in which are tens to hundreds of times higher than in "normal" elements and may have a different dependence on temperature.
\end{abstract}

\section{Keywords}

Star sensor, dark current, charge-coupled matrix device

\section{Introduction}

The problem of recalculating the star magnitudes of navigation catalogs arises when creating any sensor, since an input (source) catalog is required, complete up to a certain star magnitude in the sensitivity band of the sensor, and direct observations of stars in the photometric bands used in these instruments are usually absent. For the SAI star sensor, the recalculation problem is even more important, since during its operation this sensor uses information on the brightness of the stars. Because of this, the accuracy of calculating the magnitudes in the sensitivity band of the silicon charge-coupled device (CCD) (panchromatic band) should be high [1].

For the calculation, we used data from the Tycho-2 [2] and 2MASS [3] catalogs. Both of these catalogs cover the entire sky and exceed the limit required to create a navigation catalog, as well as have sufficient photometric accuracy. The Tycho-2 catalog contains photometric data in two visible

\footnotetext{
Proceedings of MIP Computing-V 2022: V International Scientific Workshop on Modeling, Information Processing and Computing, January 25, 2022, Krasnoyarsk, Russia

EMAIL: aozhukov@mail.ru (A Zhukov); mike@sai.msu.ru (M Prokhorov); zakhster@gmail.com (A Zakharov); ant.biryukov@gmail.com (A Biryukov); kartsan2003@mail.ru (I Kartsan); skripatchevv@inbox.ru (V Skripachev) ORCID: 0000-0002-5122-3752 (A Zhukov); 0000-0001-7659-4341 (M Prokhorov); 0000-0003-2621-4931 (A Zakharov); 0000-0001-72106988 (A Biryukov); 0000-0003-1833-4036 (I Kartsan)

(c) (i) (C) 2022 Copyright for this paper by its authors.

Use permitted under Creative Commons License Attribution 4.0
} 
bands, $B_{t}$ and $V_{t}$, and the 2MASS catalog, in three near-IR bands $J, K, H$. Due to the fact that the accuracy of measuring the brightness of bright stars in these catalogs is rather low, for such stars we used photometric data from the $W B V R$ catalog of the SAI MSU [4] and from the $V_{C} R_{C} I_{C}$ to the Kuzins catalog $[5,6]$.

\section{Construction of the reduction polynomial}

The catalog creation procedure included the following steps:

- Primary cleaning of catalogs: elimination of variables and close binaries;

- Mutual identification of catalog stars, identification and elimination of identification errors;

- Exclusion of stars with abnormal color indices. The appearance of such color indices is associated either with the anomalous properties of the star or its variability (due to nonsimultaneous brightness measurements in the bands included in the color index), or with errors in measuring the brightness of stars in at least one band. Such stars are not suitable for conversion to other spectral bands;

- The next step for calculating the brightness of stars - the central one in the procedure - is the construction of a reduction polynomial (or a system of polynomials for various calculation cases) based on the color indices of the stars. The constructed polynomial must satisfy a number of conflicting requirements: to provide small residual deviations of the stars and, at the same time, have sufficiently low degrees. The polynomial system is required when using stars for which measurements in the catalogs are not present in all photometric bands.

The reduction polynomials were constructed according to the following scheme:

Tables of model magnitudes of stars were calculated for the reaction curves of various photometric systems in the original catalogs and for the model interstellar extinction magnitude using the formula:

$$
m_{i}^{X}=-2,51 q \frac{\int E(\lambda) \varphi_{i}(\lambda) \tau^{X}(\lambda) d \lambda}{\int E_{\alpha L y r}(\lambda) \varphi_{i}(\lambda) d \lambda},
$$

where $\boldsymbol{E}(\boldsymbol{\lambda})$ - power irradiance spectral density (PISD);

$\boldsymbol{E}_{\boldsymbol{\alpha} \boldsymbol{L} \boldsymbol{y r}}$ - PISD stars;

$\boldsymbol{\alpha} \boldsymbol{L} \boldsymbol{y} \boldsymbol{r}$ - stars Vega;

$\boldsymbol{\tau}^{X}(\lambda)$ - monochromatic interstellar absorption coefficient with optical depth $X$;

$\varphi_{i}(\lambda)$ - response curve of the receiving equipment in the $i$-th spectral band.

To correctly simulate the influence of interstellar reddening, the calculations of $m_{i}^{X}$ for each star with known PISD were carried out for a random set of values of the parameter $X$.

Reducing polynomials were compiled in the form of the dependence of the magnitude of the star in the stripe of the device $Z$ on the color indices of the selected source catalog:

$$
Z-m_{l}^{0}=\sum_{n=0}^{N} \sum_{k=1}^{K} \sum_{i=k+1}^{K} a_{n i k}\left(m_{k}^{0}-m_{i}^{0}\right)^{n}
$$

where $Z$ - magnitude of the star in the stripe of the star sensor;

$m_{l}^{0}$ - the magnitude of the star in the band of the original catalog, which is closest in position in the spectrum to the $Z$ band;

$m_{k}^{0}-m_{i}^{0}$ - color indices of stars from the source catalog;

$K$ - number of bands in the photometric system of the original catalog;

$N$ - the order of the polynomial used;

$a_{\text {nik }}$ - polynomial coefficients.

The order of the polynomial was limited to $N=3$, which turned out to be quite sufficient for approximation with the required accuracy.

Using the nonlinear least squares method (the Marquardt - Levenberg method), the values of the coefficients of the polynomials $a_{\text {nik }}$.

After finding the coefficients, an analysis of their significance was carried out; insignificant coefficients were discarded. Then the next iteration was carried out. Usually 6-10 iterations are enough (depending on the number of bands in the source catalog) to determine the significant terms of the polynomials. 
In addition to model calculations performed according to this scheme, polynomials were used in which color indices from the corresponding catalogs for those stars were inserted into the right side of the formula for those stars whose $Z$ values were calculated using the known PISD. This method of constructing polynomials gives a less accurate approximation due to random and systematic errors in the magnitudes of stars in the original catalogs. On the other hand, it depends little on errors in the calibration of the PISD in the original spectrophotometric catalogs and, therefore, gives an independent estimate of the desired values of $Z$.

Another advantage of this method is the ability to compose combined polynomials from the color indicators of several catalogs containing measurements not only in the visual range, but also in the infrared. For example, in the Tycho-2 survey catalog, which contains more than 2.5 million stars [2], there is only one color index $\left(B_{T}-V_{T}\right)$, while the 2MASS survey catalog has only two: $(J-H),(H-K)[3]$. The combination of these catalogs makes it possible to obtain in this way a large number of magnitudes of stars in the $Z$ band for stars identified in both catalogs.

As an example of a photometric polynomial for determining the magnitude of a star in the $Z$ band according to $B_{T} V_{T} J H K$ :

$$
\begin{gathered}
Z=V_{T}+0,1007\left(B_{T}-V_{T}\right)-0,1167\left(V_{T}-J\right)^{2}-0,2948\left(V_{T}-J\right)(H-K)+ \\
+1,2929(J-H)(H-K)-3,8485\left(B_{T}-V_{T}\right)^{3}+0,3949\left(V_{T}-J\right)^{3}- \\
-6,1329(H-K)^{3}+5,5895\left(B_{T}-V_{T}\right)^{2}\left(V_{T}-J\right)+5,0977\left(B_{T}-V_{T}\right)^{2}(J-H)- \\
-2,6155(J-H)^{2}\left(B_{T}-V_{T}\right)+1,1965\left(V_{T}-J\right)^{2}(H-K)+0,4554\left(V_{T}-J\right)^{2}(H-K)- \\
-2,2947(J-H)^{2}\left(B_{T}-V_{T}\right)+2,6589(J-H)^{2}(H-K)+1,3181(J-H)^{2}\left(V_{T}-J\right)+ \\
+6,0792(H-K)^{2}\left(B_{T}-V_{T}\right)-2,848(H-K)^{2}\left(V_{T}-J\right)-5,0479(H-K)^{2}(J-H)- \\
-5,0380\left(B_{T}-V_{T}\right)\left(V_{T}-J\right)(J-H)-3,1368\left(B_{T}-V_{T}\right)(J-H)(H-K)
\end{gathered}
$$

The photometric characteristics were verified by comparing the $Z$ values obtained from different source catalogs. For this, a list of common stars of the $W B V R$ catalogs of the SAI MSU, Tycho-2, $U c$, $B c, V c, R c, I c$ Kuzins, UBVRI Johnson, JHK 2MASS was compiled. The prepared polynomials for these stars were used to calculate the values of $Z$. To check the reliability of the polynomials, the differences in the values of $Z$ were plotted, calculated from different sources. For example, Figure 1 and 2 show the dependences of such differences on the color index and the magnitudes of the stars.

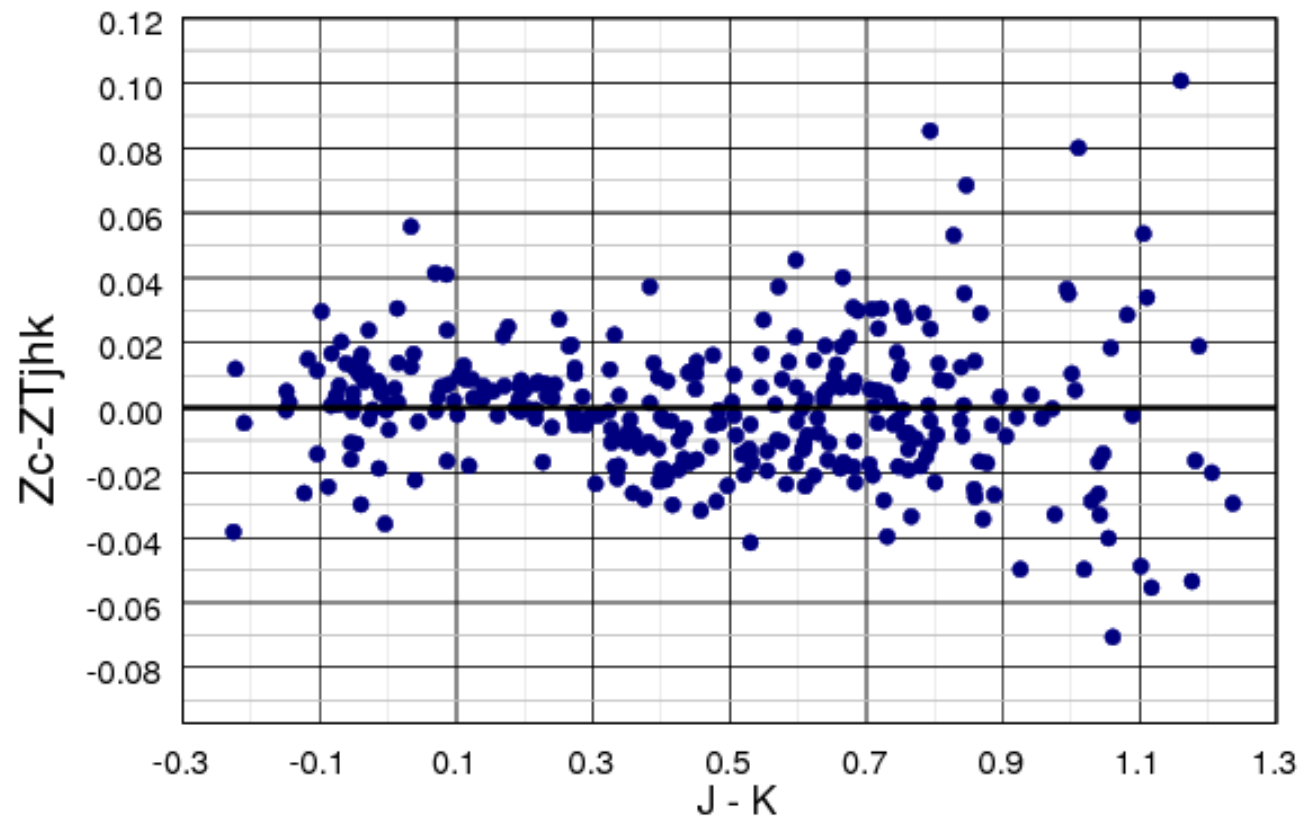

Figure 1: Dependence of the difference between the value $\mathrm{Z}_{\mathrm{c}}$, calculated according to the Kuzins catalog, and the value $\mathrm{Z}_{\mathrm{tjhk}}$, calculated according to $\mathrm{B}_{\mathrm{T}} \mathrm{V}_{\mathrm{T}} \mathrm{JHK}$, on the color index J-K of the 2MASS catalog 


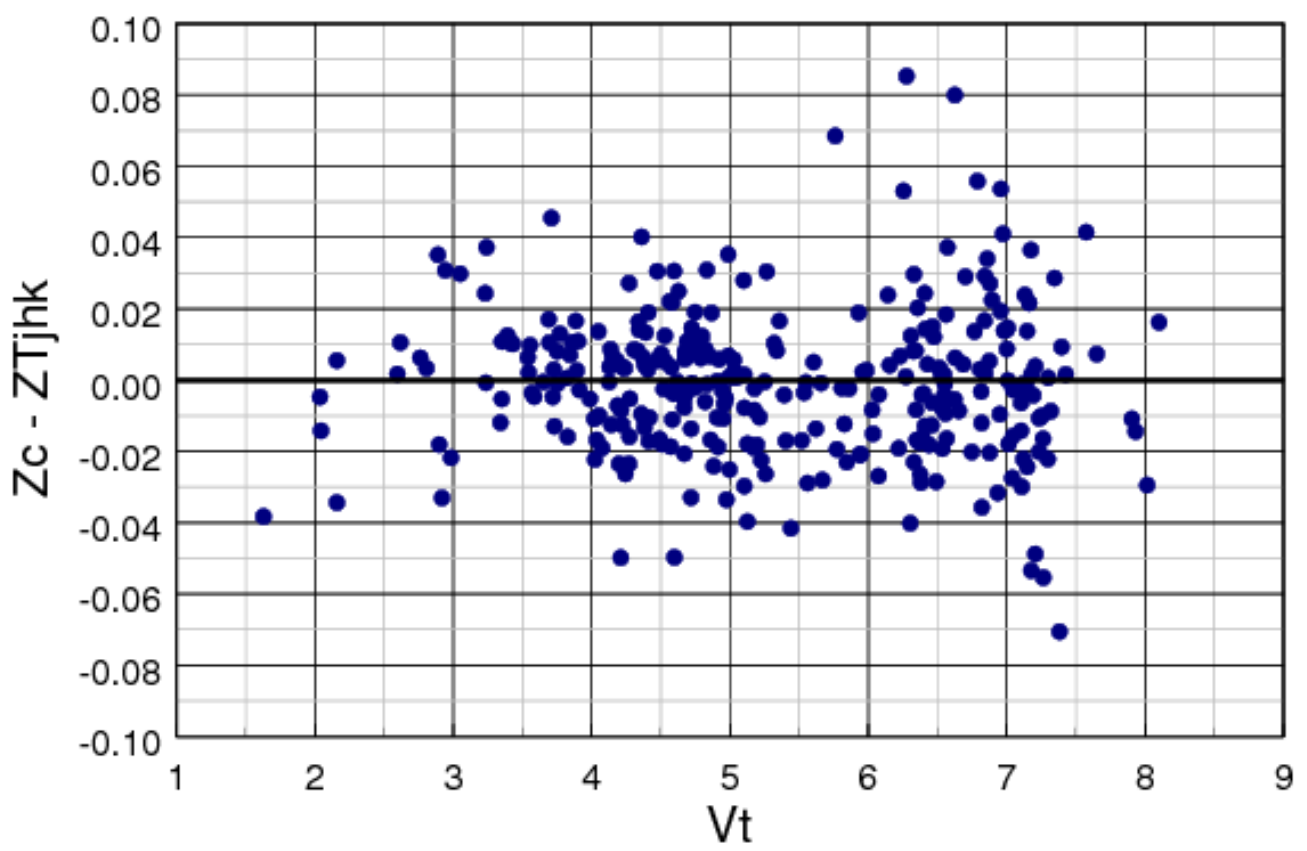

Figure 2: Dependence of the difference between the $\mathrm{Z}_{\mathrm{c}}$ value calculated according to the Kuzins catalog and the $\mathrm{Z}_{\mathrm{tjhk}}$ value calculated using the $\mathrm{B}_{\mathrm{T}} \mathrm{V}_{\mathrm{T}} \mathrm{JHK}$ on the $\mathrm{V}_{\mathrm{T}}$ value of the star in the Tycho- 2 catalog

Analysis of the graphs shows that the value of the standard deviation of the difference in magnitudes is $0.027^{\mathrm{m}}$, the spread of values slightly increases in the region of red stars. The average value for 320 common stars is $0.0018^{\mathrm{m}}$, that is, there is practically no dependence either on the color index or on the magnitude.

Similar plots have been built for other photometric catalogs. In the presence of measurements of the star in different catalogs, we took the weighted average value of $Z$.

\section{Conclusion}

The problem of recalculating the stellar magnitudes of navigational catalogs arises in the creation of any sensor, because we need an input (original) catalog, complete to a certain stellar magnitude in the sensitivity band of the sensor, and direct observations of stars in the photometric bands used in these devices are usually absent.

The presented method can be adapted to different spectral bands and input catalogs. In the work [7], an attempt was made to improve the method proposed here by compiling other types of approximating polynomials. In our opinion, the results of the study confirm the advantage of the SAI method. Also an advantage of the presented method is the possibility to compose combined polynomials from the color indices of several catalogs containing measurements not only in the visual range, but also in the infrared range.

\section{Acknowledgements}

This work was carried out within the framework of the state assignment of the Ministry of Education and Science of the Russian Federation on "Conceptual modeling of the information and educational environment of human capital reproduction in a digital economy" 121102600069-2.

This work was performed within the framework of the state assignment of the Ministry of Education and Science of Russia on the topic "Development of new methods of autonomous navigation of spacecraft in outer space" 121102600068-5.

This study was supported by the Russian Federation State Task No 0555-2021-0005. 


\section{References}

[1] O. I. Stekolschikov, A. I. Zakharov, M. E. Prokhorov, Design Philosophy of a Star Tracker of the SAI MSU with the Mirror Objective and Narrow Field of View, Mechanics, Control and Informatics (2013) 69-79.

[2] The Hipparcos and Tycho Catalogues, ESA SP-1200, ESA, 1997.

[3] R. M. Cutri, M. F. Skrutskie, Dyk S. Van, C. A. Beichman, J. M. Carpenter, T. Chester, L. Cambresy, T. Evans, J. Fowler, J. Gizis, E. Howard, J. Huchra, T. Jarrett, E. L. Kopan, J. D. Kirkpatrick, R. M. Light, K. A. Marsh, H. McCallon, S. Schneider, R. Stiening, M. Sykes, M. Weinberg, W. A. Wheaton, S. Wheelock, N. Zacarias, The 2MASS All-Sky Catalog of Point Sources, The IRSA 2MASS All-Sky Point Source Catalog, NASA/IPAC Infrared Science Archive, URL: http://irsa.ipac.caltech.edu/applications/Gator/, 2003.

[4] V. G. Kornilov, I. M. Volkov, A. I. Zakharov, V. S. Kozyreva, L. N. Kornilova, A. N. Krutyakov, A. V. Krylov, A. V. Kusakin, S. Ye. Leont'yev, A. V. Mironov, V. G. Moshkalev, T. M. Pogrosheva, V. N. Sementsov, KH. F. Khaliullin, Catalog of WBVR-values of bright stars in the northern sky, Proceedings of the SAI MSU, 1991.

[5] M. W. Richmond, T. F. Droege, G. Gombert, M. Gutzwiller, A. A. Henden, C. Albertson, N. Beser, N. Molhant, H. Johnson, TASS Mark III photometric survey, The Publications of the Astronomical Society of the Pacific 112 (2000) 397-408.

[6] V. S. Averyanov, I. N. Kartsan, S. V. Efremova, Methods of automated detection of anomalies and nonlinear transitions by autonomous unmanned aerial vehicles Journal of Physics: Conference Series 1889(4) (2021) 042001. doi: 10.1088/1742-6596/1889/4/042001.

[7] I. S. Kruzhilov, Evaluation of instrument star magnitudes without recourse to data as to star spectral classes, Journal of Applied Remote Sensing 6 (2012) 063537. 